\title{
ACLIMATACION DE Uncaria tomentosa (Willd.) DC. PRODUCIDA IN VITRO
}

\section{ACLIMATIZATION OF Uncaria tomentosa (Willd.) DC. PRODUCED IN VITRO}

\author{
Gilberto Domínguez Torrejón ${ }^{1}$ y María Luz Donayre Gómez ${ }^{2}$
}

\begin{abstract}
Resumen
Ensayos previos de aclimatación de vitroplantas de Uncaria tomentosa (Willd.) DC, (Uña de gato), determinaron la susceptibilidad de la especie a adaptarse a condiciones de ambiente natural. Se seleccionó un clon con la mayor tasa de multiplicación in vitro, para su evaluación en la fase de aclimatación y se le sometió a mediciones de supervivencia, crecimiento en altura y vigorosidad expresado en número de hojas desarrolladas en esta fase. Los resultados ha permitido obtener el $100 \%$ de supervivencia, con tamaños de vitroplantas entre $17.07 \mathrm{~cm}$ y $19.53 \mathrm{~cm}$ en el periodo de pre aclimatación y con una vigorosidad entre 7 y 14 hojas aproximadamente; características que no han tenido ninguna incidencia en la mortandad. Desarrollo posterior en diferentes substratos, no mostró diferencias significativas en el tamaño de las plantas $(20.8-24.8 \mathrm{~cm})$, por lo que se las considera como de tamaño homogéneo; a pesar de haberse notado un ligero efecto de menor incremento en uno de los substratos. La vigorosidad final de las vitroplantas expresada en número de hojas tampoco mostró diferencias significativas por efecto de los substratos, por lo que se las considera como plantas de vigor homogéneo para ser destinadas al campo.
\end{abstract}

Palabras claves: Aclimatación, Uncaria tomentosa, clon, vitroplantas

\begin{abstract}
Previous acclimatization assays of Uncaria tomentosa ( Willd.) DC. (cat's claw) in vitro plants, determined the susceptibility of the species to adapt to natural environment conditions. An in vitro created clone was selected for evaluation in the acclimatization phase and it was subjected to survival, growth and level of strength measurements, expressed in number of leaves developed. The results show that the acclimatization process has permitted a rate of $100 \%$ survival of in vitro plants, with sizes from $17.07 \mathrm{~cm}$ to $19.53 \mathrm{~cm}$ in the pre acclimatization period, with a level of strength of 7 to 14 leaves approximately. The figures show that these have not had incidence in the mortality. The delayed development of in vitro plants in several substrata did not show significant differences in the size of the plants $(20.8-24.8 \mathrm{~cm}$.). Despite of having a slight effect of lower increase in one of the substrata, their size is considered homogeneous. The final strength of in vitro plants, expressed in number of leaves did not show significant differences due to substrata, allowing these plants of homogeneous vigor to be considered suitable to be used in the fields.
\end{abstract}

Key words: aclimatization, Uncaria tomentosa, clon, vitroplant

\section{Introducción}

El establecimiento de plantaciones para la producción de materia prima con calidad controlada y en menores espacios territoriales, requiere de un proceso de domesticación ordenado, con material genético conocido que garantice la calidad de la materia prima. Para ello, el cultivo in vitro de esta especie es una alternativa interesante, por la necesidad de masificar la producción con la seguridad de contar con productos de calidad homogénea. Esta tecnología se ha venido desarrollando en la Universidad Agraria La Molina (UNALM) desde el año 1993, lográndose la estandarización de la producción in vitro de Uncaria tomentosa (Domínguez \& Tapia, 2000). Diversos factores no facilitaron en su momento realizar ensayos de aclimatación de esta especie con fines productivos por lo que en el presente estudio se demuestra la factibilidad de esta etapa de la micropropagación.
La propagación por semillas es uno de los métodos convencionales que mejores resultados reportan varios autores como método de propagación de Uncaria tomentosa. Para ello la recolección de semillas debe realizarse oportunamente antes de su diseminación, ya que este es un proceso violento que por su forma alada y su tamaño minúsculo (un gramo de semillas equivale a 5 000-7 000 unidades), dificulta las labores de cosecha (Flores, 1995).

Experimentos de propagación por estacas de Uncaria tomentosa se han iniciado con mayor incidencia en el año 1995, aunque no necesariamente se han considerado sistemáticamente las variables que pueda incidir en el éxito del enraizamiento, como: tipo de estaca, condición fisiológica de la planta madre, edad de la planta madre, condiciones ambientales, aplicación de sustancias hormonales, etc. (Domínguez, 1997). Trabajos sobre propagación por estacas realizados en el Proyecto Especial Alto Huallaga 
(Cuellar, 1996), demuestran solo formación de callos, proliferación de brotes o bajos porcentajes de enraizamiento.

En la propagación in vitro, de acuerdo al tipo de material vegetal utilizado para su introducción a estas condiciones, se pueden resumir tres tipos de tecnología: El cultivo de órganos (yemas axilares y apicales, nudos de talluelos), el cultivo de tejidos (hojas, peciolos, meristemas) y el cultivo de células (células y protoplastos). En todos los casos se puede lograr como producto final plantas enraizadas que van a ser sometidas a un proceso de aclimatación, previamente a la etapa de desarrollo en viveros convencionales, en donde lograrán el crecimiento necesario para su instalación en campo definitivo (Domínguez, 1997).

Los Avances logrados en este aspecto con Uncaria tomentosa en el Laboratorio de Estudios biológicos de Germoplasma de la UNALM, se refieren a dos tipos de explantes utilizados como material inicial, semillas germinadas in vitro y fragmentos de hojas. En ambos casos se ha logrado desarrollar plantas completas y vigorosas que ha permitido realizar un proceso de selección fenotípica basada en las características morfológicas de las microplantas (número de hojas, tamaño de hojas, largo de talluelo, largo de entrenudo, largo de raíces, vigorosidad de raíces) (Domínguez, 1997).

La aclimatación es la etapa final necesaria en todos los esquemas de micro propagación. Las plantas deben adaptarse a nuevas condiciones ambientales tales como, baja humedad relativa, alta intensidad de luz, fluctuaciones de temperatura y constante estrés de resistencia a enfermedades. La calidad intrínseca de las plántulas in vitro es uno de los mas importantes factores que gobierna estos sucesos durante la transición a ex vitro. Son de ellos la excesiva pérdida de agua por transpiración y un debilitado aparato fotosintético los mayores problemas que se tiene que superar (Luya, 1999).

El mayor porcentaje de pérdidas de plantas producidas in vitro ocurren en su fase de transferencia al suelo cuando deben adaptarse a las nuevas condiciones del ambiente edáfico (Roca, 1991). Casi todo el esfuerzo investigativo del cultivo de tejidos se perderían si las plantas que se regeneran murieran cuando se intenta desarrollarlas en un ambiente que en un comienzo les resulta desfavorable.

El éxito de la propagación in vitro radica en lograr la aclimatación de las vitroplantas a las condiciones ambientales. Durante esta etapa se produce un retorno gradual al funcionamiento autotrófico de las vitroplantas, así como la recuperación de las características morfológicas y fisiológicas normales, asimismo en esta etapa las plántulas sufren un estrés provocado por al cambio de las condiciones de humedad y temperatura, por lo que la transferencia debe de realizarse de forma gradual (Sotolongo, 2000).

La supervivencia de las vitroplantas regeneradas durante el periodo de adaptación depende fundamentalmente de las peculiaridades fisiológicas, estructurales y anatómicas que las plántulas presentan producto del desarrollo in vitro, lo cual permite una elevada humedad relativa en el interior de los frascos, baja intensidad luminosa, bajo intercambio gaseoso, abundante disponibilidad de nutrientes y carbono (generalmente en forma de sacarosa) y una pequeña variación de temperatura en un rango considerado óptimo para el cultivo (Preece \& Sutter, 1991; Teixeira et al., 1995).

\section{Metodología}

Materiales y equipos

Se utilizaron plántulas de Uncaria tomentosa obtenidas a partir de la micropropagación de yemas provenientes de material generativo en el laboratorio del Instituto de Biotecnología de la UNALM en Lima. Este material fue seleccionado clonalmente desde su iniciación considerando características morfológicas en su desarrollo.

Las plántulas con un promedio de un mes y medio en condiciones de laboratorio, fueron trasladadas en frascos de vidrio en sus propios medios de cultivo al laboratorio de biotecnología de la Universidad Nacional de Ucayali (UNU) en Pucallpa-Ucayali, en donde posteriormente fueron transferidas a 4 sustratos de tierra:arena:humus (3:2:1) diferenciándose en la procedencia de la tierra proveniente de 4 zonas edáficas de la Regiòn Ucayali (Tramo de 203 km de la carretera Pucallpa-Tingo Maria - Figura 1).

El sustrato fue colocado en envases individuales de plástico transparente los que fueron instalados en el invernadero de la UNU. Para su control y transporte se utilizaron: termómetro, regadera, carretilla y materiales de codificación.

\section{Procedimiento}

Las plantas fueron transportadas de Lima a Pucallpa en el mes de diciembre. Diez días después se hicieron agujeros en la cobertura de los frascos que contenían las plántulas, ubicados en la sala de incubación del laboratorio de la UNU, dos días después fueron trasladadas al invernadero en donde se realizó, al día siguiente, el transplante a los vasos de pre aclimatación, previamente llenados con el sustrato correspondiente. Los envases conteniendo la planta fueron cubiertos con otro envase del mismo tamaño, los mismos que fueron gradualmente aperturados mediante agujeros en la cobertura hasta dejar la planta completamente expuesta al ambiente natural del invernadero a una temperatura promedio de $35^{\circ} \mathrm{C}$, después de cuatro semanas de pre aclimatación. Dos días después fueron transferidas a bolsas de polietileno, manteniendo los mismos sustratos para darles un mayor espacio de desarrollo radicular, en 
donde se aclimataron durante cuatro semanas, antes de llevarlas a vivero.

\section{Evaluaciones}

Se evaluó la supervivencia de las vitroplantas y se hicieron mediciones de altura y numero de hojas por planta tomándose al azar 30 plantas por cada sustrato. simple, a través del paquete estadístico Statistical Package for Social Science (SPSS).

\section{Resultados y discusión}

\section{Sobrevivencia de vitroplantas}

Se determinó la influencia del sustrato en la supervivencia y vigorosidad de la planta expresada en

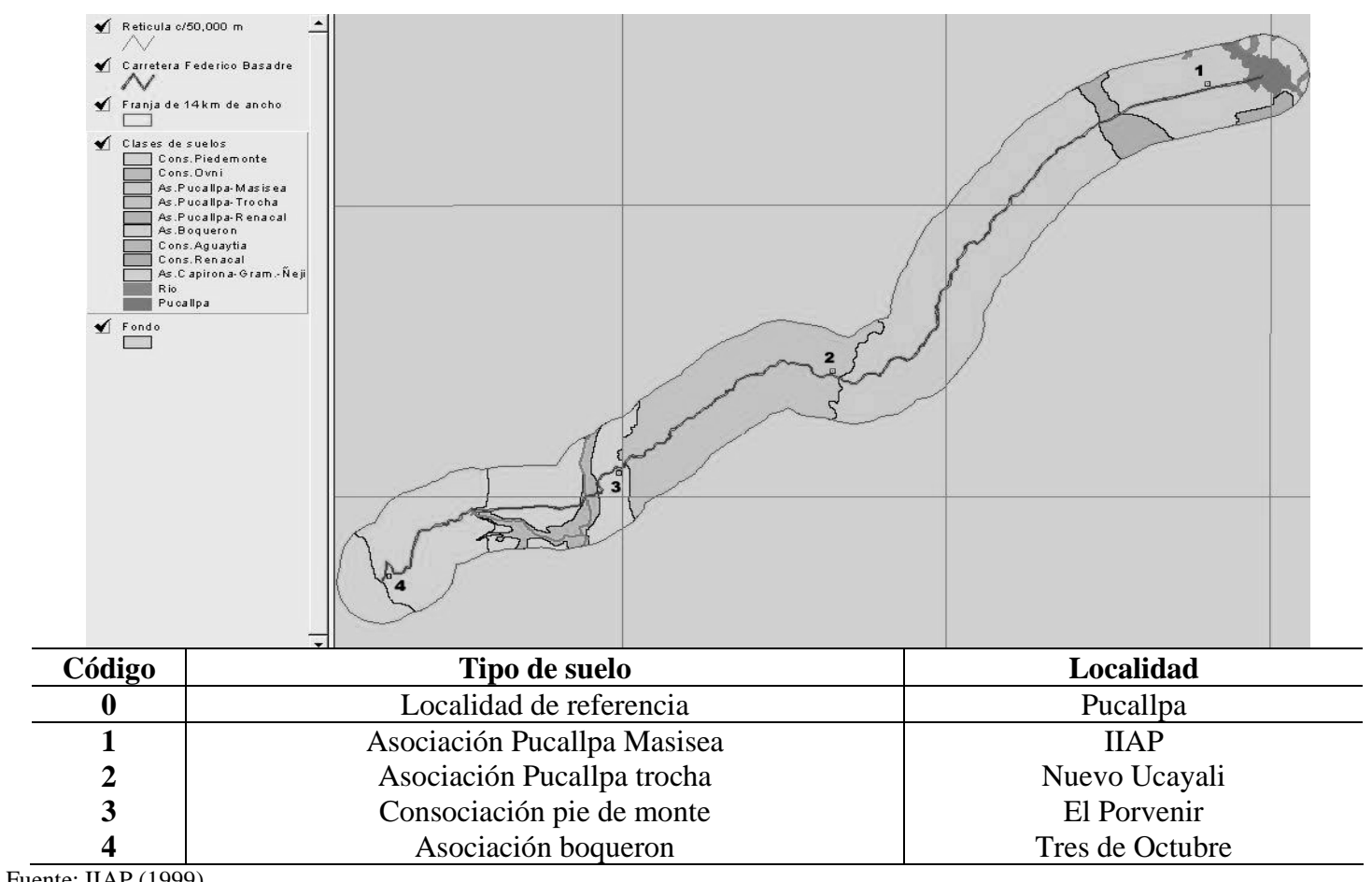

Fuente: IIAP (1999)

Figura 1: Procedencia de tierra para substratos

Se hicieron 4 mediciones posteriores a la pre aclimatación en un periodo de 30 días, época en que las plantas estaban listas para su traslado a condiciones de trasplante a bolsas y crecimiento en vivero

$>$ Variables en Estudio

X1 : Altura al inicio de la pre aclimatación

X2 : Número de hojas al inicio de la pre aclimatación

X3 : Altura de la planta al término de la aclimatación

X4 : Número de hojas desarrolladas por la planta al término de la aclimatación

X5 : Incremento en la altura de la planta en la fase de aclimatación

Análisis biométrico:

Cada substrato representa un tratamiento y las vitroplantas fueron instaladas bajo un diseño completamente al azar, con análisis de varianza altura y número de hojas, así como también se validó parámetros micro ambientales durante el proceso de aclimatación. La mortandad de vitroplantas en esta etapa es un factor de alto riesgo si no se tiene establecido las condiciones adecuadas para este proceso de adaptación y el estrés de cambio puede afectar en pocas horas la supervivencia de la planta. Las plantas producidas en estas condiciones pueden resultar de fácil aclimatación, mientras que en otras los déficit morfológicos y funcionales dificultarán tremendamente el trasplante y posterior aclimatación. Es decir como consecuencia del ambiente in vitro las vitroplantas son incapaces de sobrevivir al trasplante directo al invernadero o campo (Reigosa et al., 2004). En el caso de Uncaria tomentosa la susceptibilidad al cambio de humedad microambiental y temperatura entre el espacio cerrado de desarrollo dentro del frasco de vidrio y el espacio de desarrollo en transición al ambiente natural, ha sido evidente. Ensayos preliminares realizados fuera de su ámbito de desarrollo natural demostraron tasas de mortalidad 
entre 40 a $60 \%$ en un ambiente de invernadero de la UNALM, ubicado en Lima (costa central del Perú), mientras que los mismos ensayos realizados en el invernadero de la UNU utilizando diferentes clones producidos en el laboratorio de biotecnología de la UNALM han alcanzado, en todos los substratos, tasas de supervivencia de $100 \%$, de manera que el tipo de substrato no ha sido un factor determinante de la supervivencia de plantas durante la aclimatación.

2. Análisis de las condiciones de las vitro plantas al final de la pre aclimatacion

Las condiciones en las que se llevó a cabo esta etapa de pre aclimatación, además de los substratos utilizados y mencionados en la metodología, fueron a una temperatura promedio de invernadero de $35^{\circ} \mathrm{C}$ durante el día y $90 \%$ de humedad relativa micro ambiental dentro de los ambientes individuales temporalmente cerrados.

> Análisis Descriptivo de la altura inicial

En términos promedios, no se distinguen diferencias significativas en la altura de la planta al inicio de la aclimatación (Figura 2); sin embargo, en el substrato de la localidad de El Porvenir se presenta la máxima altura inicial promedio (19.53 cm) por planta, mientras que la mínima altura inicial promedio se da para el substrato de la localidad km 203 (17.07 cm) (Tabla 1).

Tabla 1. Estadísticas Descriptivas de la Altura Inicial.

\begin{tabular}{lcccccc}
\hline & $\begin{array}{c}\mathbf{N}^{\mathbf{d}} \mathbf{d e} \\
\text { datos }\end{array}$ & Mínimo & Máximo & Promedio & $\begin{array}{c}\text { Desviación } \\
\text { Estándar }\end{array}$ & CV \\
\hline IIAP & 30 & 13 & 25.00 & 17.9333 & 4.39383 & 24.50 \\
Km 98 & 30 & 12 & 25.00 & 18.4667 & 4.11669 & 22.29 \\
Porvenir & 30 & 12 & 27.00 & 19.5333 & 4.27288 & 21.88 \\
Km 203 & 30 & 12 & 23.00 & 17.0667 & 4.02521 & 23.58 \\
$\begin{array}{l}\text { Valid N } \\
\text { (Listwise) }\end{array}$ & 30 & & & & & \\
\hline
\end{tabular}

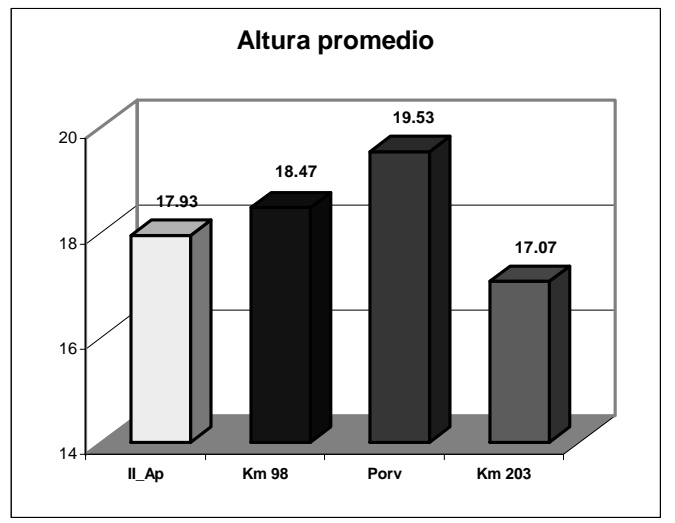

Figura 2. Altura promedio de vitroplantas al final de la pre aclimatación.

Estas diferencias, que no son significativas, se consideran normales en vitroplantas y son debidas a los distintos factores de estrés que tienen que soportar durante las fases de la micropropagación; Reigosa et al. (2004), mencionan al respecto que todas las plantas producidas en recipientes con escaso intercambio gaseoso, pueden manifestar alteraciones o déficit en cuanto a su estructura anatómica, morfológica y fisiológica dependiendo de la especie e incluso de la variedad.

En términos de variabilidad en la altura de la planta, se distingue una menor dispersión de este parámetro en las vitroplantas instaladas en el substrato El Porvenir; la mediana en este substrato, con las del substrato km 98 son muy cercanas. Las alturas de vitroplantas instaladas en los substratos, Instituto de Investigaciones de la Amazonía Peruana (IIAP), Km 98 y Km 203, están aproximadamente en un mismo rango (entre 13 y $22 \mathrm{~cm}$ ).

Tabla 2. Intervalo de confianza para la Altura inicial promedio.

\begin{tabular}{|c|c|c|c|c|c|c|}
\hline & \multicolumn{6}{|c|}{ Test Value $=0$} \\
\hline & \multirow[b]{2}{*}{$\mathbf{t}$} & \multirow[b]{2}{*}{ Df } & \multirow{2}{*}{$\begin{array}{c}\text { Sig. } \\
\text { (2- } \\
\text { tailed) }\end{array}$} & \multirow{2}{*}{$\begin{array}{l}\text { Mean } \\
\text { Difie } \\
\text {-rence }\end{array}$} & \multicolumn{2}{|c|}{$\begin{array}{l}\text { 95\% Confidence } \\
\text { Interval of the } \\
\text { Difference } \\
\end{array}$} \\
\hline & & & & & Lower & Upper \\
\hline IIAP & 22.355 & 29 & .000 & 17.93333 & 16.2927 & 19.5740 \\
\hline Km 98 & 24.570 & 29 & .000 & 18.46667 & 16.9295 & 20.0039 \\
\hline Porvenir & 25.039 & 29 & .000 & 19.53333 & 17.9378 & 21.1289 \\
\hline Km 203 & 23.223 & 29 & .000 & 17.06667 & 15.5636 & 18.5697 \\
\hline
\end{tabular}

Con un nivel de confianza del $95 \%$ podemos afirmar que la altura inicial promedio poblacional en el substrato de El Porvenir se encuentra entre $17.94 \mathrm{~cm}$ y $21.13 \mathrm{~cm}$ y es superior a las obtenidas en los demás substratos (Tabla 2). Mientras que el intervalo de confianza obtenido en los substratos de IIAP y el Km 98 son similares. Por otro lado, se observa que en todos los substratos la amplitud interválica es aproximadamente $3.5 \mathrm{~cm}$.

Análisis Descriptivo del número inicial de hojas Analizando el número promedio de hojas desarrolladas por las vitroplantas al inicio de la aclimatación (Tabla 3), se observa que en aquellas instaladas en el substrato El Porvenir, se presenta el promedio más alto (aprox. 12 hojas), a diferencia de las vitroplantas del substrato Km 203 en donde se tiene el mínimo número de hojas (aprox. 9), esto es correspondiente con las diferencias de altura entre ambos. En el caso de vitroplantas instaladas en substratos IIAP y Km 98, este último tiene un menor promedio de número de hojas (1.3 de diferencia), a pesar de que su altura promedio es ligeramente superior $(0.54 \mathrm{~cm})$. 
Tabla 3. Estadísticas Descriptivas del Número de Hojas.

\begin{tabular}{lcccccc}
\hline & $\begin{array}{c}\mathbf{N}^{\mathbf{0}} \text { de } \\
\text { datos }\end{array}$ & Mínimo & Máximo & Promedio & $\begin{array}{c}\text { Desviación } \\
\text { Estandar }\end{array}$ & $\mathbf{C V}$ \\
\hline IIAP & 30 & 6.00 & 22.00 & 10.8000 & 3.37741 & 31.27 \\
Km 98 & 30 & 4.00 & 16.00 & 9.5000 & 3.57915 & 37.68 \\
Porvenir & 30 & 7.00 & 28.00 & 12.5333 & 4.45236 & 35.61 \\
Km 203 & 30 & 4.00 & 20.00 & 8.9667 & 3.02271 & 33.71 \\
Valid N & 30 & & & & & \\
(Listwise) & & & & & & \\
\hline
\end{tabular}

hojas de las vitroplantas transferidas a los 4 sustratos no son significativos para iniciar un proceso de aclimatación, en la medida en que experiencias preliminares han demostrado una alta supervivencia en sustratos estériles con tamaños de planta similares, determinados por el tiempo de permanencia de las mismas en condiciones de laboratorio (6 semanas), antes de su salida a la fase de aclimatación

Desarrollo de las plantas durante la aclimatación.

Las características fisiológicas y anatómicas de las plantas obtenidas en recipientes con baja tasa de intercambio gaseoso hacen imprescindible realizar una aclimatación gradual al ambiente ex vitro, convirtiéndose esta parte del proceso, conjuntamente con el costo de producción, en el principal factor limitante de la micropropagación (Reigosa et al., 2004).

Las técnicas más eficaces durante esta fase, son los que van encaminadas a lograr gradualmente menos humedad relativa, más luz, crecimiento autotrófico y un medio séptico (Agramonte, 1998). En ese sentido la técnica de control individual de las vitroplantas de Uncaria en los primeros días de transferencia a substrato sólido desinfectado, dio un resultado 100\% satisfactorios; en estas condiciones se mantuvieron con una alta humedad relativa evitando el exceso de transpiración de las plantas hasta que logren un adecuado desarrollo de los estomas y la cutícula, como lo manifiesta Ziv (1991).

La aclimatación de vitroplantas de Uncaria tomentosa se logró en un periodo de ocho semanas, correspondiendo las cuatro primeras semanas a un periodo de pre aclimatación. En esta etapa, se tomó en cuenta la temperatura y la humedad relativa al interior de los envases de cobertura, como los factores de mayor importancia. Estos fueron manejados a través de perforaciones graduales de la cobertura hasta su retiro total y adaptación a las condiciones del ambiente de invernadero.

Dos días después las plántulas fueron transferidas a bolsas conteniendo los mismos substratos originales a fin de darles mayor espacio radicular y facilitar a su vez el transporte a campo definitivo. En estas condiciones se inició las mediciones semanales por un periodo de un mes hasta lograr tallos lignificados.

$>$ Análisis Descriptivo de la altura final

Con un nivel de confianza del 95\% podemos

Tabla 5. Estadísticas Descriptivas de la Altura Final de la Planta.

afirmar que el número promedio de hojas en las plantas del substrato El Porvenir, se encuentra entre 11 y 14 aproximadamente, y es superior a las vitroplantas de los demás substratos (Tabla 4). Se observa además que el intervalo de confianza en los substratos de Km 98 y el Km 203 son similares.

En general se puede decir que las diferencias iniciales de tamaño y número de

\begin{tabular}{lcccccc}
\hline & $\begin{array}{c}\mathbf{N}^{\mathbf{d}} \mathbf{d e} \\
\text { datos }\end{array}$ & Mínimo & Máximo & Promedio & $\begin{array}{c}\text { Desviación } \\
\text { Estandar }\end{array}$ & CV \\
\hline IIAP & 30 & 15.00 & 27.00 & 20.8000 & 4.42875 & 21.29 \\
Km 98 & 30 & 15.00 & 28.00 & 22.7667 & 4.76107 & 20.91 \\
El & 30 & 16.00 & 30.00 & 24.8000 & 4.29434 & 17.31 \\
Porv & & & & & & \\
Km & 30 & 14.00 & 28.00 & 21.6333 & 4.63483 & 21.42 \\
$\mathbf{2 0 3}$ & & & & & & \\
\hline
\end{tabular}


Diciembre 2006

En términos promedios, se observa que las vitroplantas del substrato El Porvenir mantienen la predominancia en altura final $(24.80 \mathrm{~cm})$ lograda por la planta después de cuatro semanas de crecimiento (Tabla 5). Esto no sucede de igual forma con las vitrolantas del substrato IIAP que logran alcanzar la mínima altura final de los cuatro substratos evaluados $(20.80 \mathrm{~cm})$. Es decir que se puede aseverar que ha habido una influencia de este substrato en el desarrollo promedio de las vitroplantas en la fase de aclimatación. Por el contrario las vitroplantas del substrato Km 203, que inicialmente tenían el promedio de altura mas bajo, han logrado un mayor desarrollo que las anteriores.

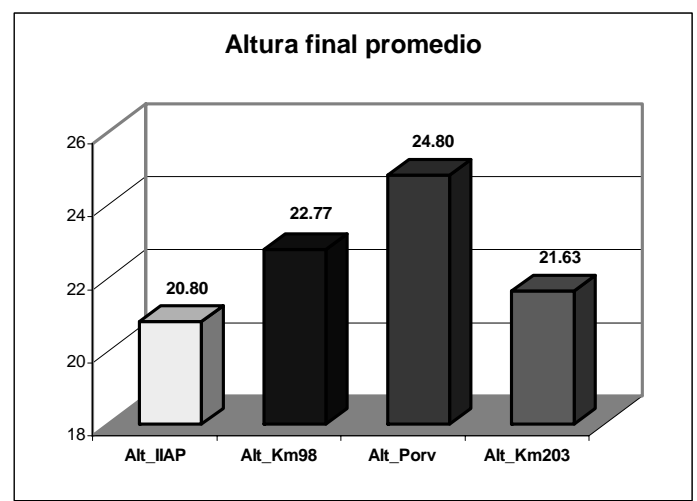

Figura 4. Altura de vitroplantas al final del periodo de aclimatación.

En términos de variabilidad en la altura de la planta obtenida en las ocho semanas, no se distingue una diferencia significativa; no obstante, la ubicación de la mediana indica que en los substratos El Porvenir y IIAP se presentan la máxima y mínima altura, respectivamente.

Tabla 6. Intervalo de Confianza para la Altura Final Promedio.

\begin{tabular}{|c|c|c|c|c|c|c|}
\hline & \multicolumn{6}{|c|}{ Test Value $=0$} \\
\hline & \multirow[b]{2}{*}{$\mathbf{T}$} & \multirow[b]{2}{*}{ df } & \multirow{2}{*}{$\begin{array}{c}\text { Sig. } \\
\text { (2-tailed) }\end{array}$} & \multirow{2}{*}{$\begin{array}{c}\text { Mean } \\
\text { Differen } \\
\text { ce } \\
\end{array}$} & \multicolumn{2}{|c|}{$\begin{array}{c}\text { 95\% Confidence } \\
\text { Interval of the } \\
\text { Difference }\end{array}$} \\
\hline & & & & & Lower & Upper \\
\hline IIAP & 25.724 & 29 & .000 & 20.80000 & 19.1463 & 22.4537 \\
\hline Km 98 & 26.191 & 29 & .000 & 22.76667 & 20.9889 & 24.5445 \\
\hline $\begin{array}{l}\text { Porve } \\
\text { nir }\end{array}$ & 31.631 & 29 & .000 & 24.80000 & 23.1965 & 26.4035 \\
\hline $\begin{array}{l}\text { Km } \\
203 \\
\end{array}$ & 25.565 & 29 & .000 & 21.63333 & 19.9027 & 23.3640 \\
\hline
\end{tabular}

Con un nivel de confianza del $95 \%$ podemos afirmar que la altura promedio poblacional obtenido al final de la aclimatación en el substrato de El Porvenir se encuentra entre $23.19 \mathrm{~cm}$ y $26.40 \mathrm{~cm}$ y es superior a las obtenidas en las substratos (Tabla 6). Mientras que el intervalo de confianza obtenido en los substratos de IIAP y el KM203 son similares.

> Análisis Descriptivo para el incremento en la altura final de la planta (Comparación entre la altura obtenida en la 1ra evaluación y en la 4ta valuación).

Tabla 7. Estadísticas Descriptivas del Incremento en la Altura

\begin{tabular}{lcccccc}
\hline & $\begin{array}{c}\mathbf{N}^{\text {o }} \text { de } \\
\text { datos }\end{array}$ & Mínimo & Máximo & $\begin{array}{c}\text { Prome } \\
\text { dio }\end{array}$ & $\begin{array}{c}\text { Desviación } \\
\text { Estandar }\end{array}$ & CV \\
\hline IIAP & 30 & 1.00 & 5.00 & 2.8667 & 0.81931 & 28.58 \\
Km 98 & 30 & 2.00 & 7.00 & 4.3000 & 1.26355 & 29.38 \\
Porv & 30 & 3.00 & 9.00 & 5.2667 & 1.81817 & 34.50 \\
Km & 30 & 1.00 & 8.00 & 4.5667 & 1.67504 & 36.68 \\
$\mathbf{2 0 3}$ & & & & & &
\end{tabular}

Obteniendo el incremento en la altura, en términos de la primera y última evaluación según substrato, notamos en la figura 4 que se mantiene la tendencia de crecimiento. Así se observa que en las vitroplantas del substrato El Porvenir se presenta el mayor incremento (5.27 cm.); mientras que el mínimo incremento se da en la localidad IIAP $(2.87 \mathrm{~cm})$ (Tabla 7). Cabe mencionar que en el substrato IIAP hay una observación cuyo incremento es diferente a los obtenidos en las diferentes plantas (“outlier”).

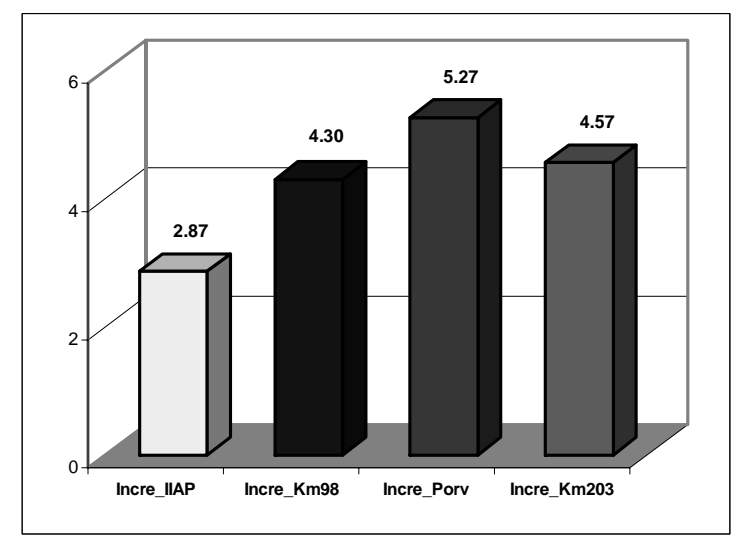

Figura 5. Promedio del incremento de la altura.

Con un nivel de confianza del $95 \%$ podemos afirmar que el incremento medio en la altura de las vitroplantas obtenido en los substratos El Porvenir $(4.59,5.94)$ y Km 203, $(3.94,5.19)$ son mayores a las alcanzadas en los otros dos substratos. Mientras que el menor intervalo de confianza se observa en el substrato de IIAP $(2.56,3.17)$ (Tabla 8).

$>$ Análisis Descriptivo del número de hojas Estadísticas Descriptivas del Número de Hojas desarrolladas por la Planta

Analizando el Número de hojas desarrolladas por la planta al final de la aclimatación, no se distinguen diferencias significativas; sin embargo, en el substrato 
El Porvenir se presenta el mayor valor (16.37 hojas), a distinción del substrato Km 203, en donde se presenta un valor mínimo de número de hojas (12.08) (Tabla 9).

Tabla 8. Intervalo de Confianza para el Incremento en la Altura.

\begin{tabular}{|c|c|c|c|c|c|c|}
\hline & \multicolumn{6}{|c|}{ Test Value $=0$} \\
\hline & \multirow[b]{2}{*}{$\mathbf{T}$} & \multirow[b]{2}{*}{ df } & \multirow{2}{*}{$\begin{array}{l}\text { Sig. } \\
(2- \\
\text { tailed) }\end{array}$} & \multirow{2}{*}{$\begin{array}{c}\text { Mean } \\
\text { Differe } \\
\text { nce }\end{array}$} & \multicolumn{2}{|c|}{$\begin{array}{c}95 \% \text { Confidence } \\
\text { Interval of the } \\
\text { Difference }\end{array}$} \\
\hline & & & & & Lower & Upper \\
\hline IIAP & 19.164 & 29 & .000 & 2.86667 & 2.5607 & 3.1726 \\
\hline Km 98 & 18.640 & 29 & .000 & 4.30000 & 3.8282 & 4.7718 \\
\hline Porvenir & 15.866 & 29 & .000 & 5.26667 & 4.5878 & 5.9456 \\
\hline Km 203 & 14.933 & 29 & .000 & 4.56667 & 3.9412 & 5.1921 \\
\hline
\end{tabular}

observa además que el intervalo de confianza obtenido en los substratos de Km 98 y el Km 203 son similares; sin embargo los coeficientes de variación hacen notar una diferencia no significativa (Tabla 10).

Tabla 10. Intervalo de Confianza para el Numero promedio de hojas.

\begin{tabular}{|c|c|c|c|c|c|c|}
\hline & \multicolumn{6}{|c|}{ Test Value $=0$} \\
\hline & \multirow[b]{2}{*}{$\mathbf{t}$} & \multirow[b]{2}{*}{ df } & \multirow{2}{*}{$\begin{array}{l}\text { Sig. } \\
\text { (2-tailed) }\end{array}$} & \multirow{2}{*}{$\begin{array}{c}\text { Mean } \\
\text { Differen } \\
\text { ce } \\
\end{array}$} & \multicolumn{2}{|c|}{$\begin{array}{c}95 \% \text { Confidence } \\
\text { Interval of the } \\
\text { Difference } \\
\end{array}$} \\
\hline & & & & & Lower & Upper \\
\hline IIAP & 18.286 & 29 & .000 & 14.16667 & 12.5822 & 15.7511 \\
\hline Km 98 & 14.458 & 29 & .000 & 13.03333 & 11.1897 & 14.8770 \\
\hline Porvenir & 16.994 & 29 & .000 & 16.36667 & 14.3969 & 18.3364 \\
\hline Km 203 & 17.626 & 29 & .000 & 12.80000 & 11.3148 & 14.2852 \\
\hline
\end{tabular}

\section{Conclusiones}

Tabla 9. Estadística descriptiva del número de hojas.

\begin{tabular}{lcccccc}
\hline & $\begin{array}{c}\mathbf{N}^{\mathbf{0}} \text { de } \\
\text { datos }\end{array}$ & $\begin{array}{c}\text { Míni- } \\
\text { mo }\end{array}$ & $\begin{array}{c}\text { Máxi } \\
\text { mo }\end{array}$ & Promedio & $\begin{array}{c}\text { Desvia- } \\
\text { ción } \\
\text { Están-dar }\end{array}$ & CV \\
\hline IIAP & 30 & 9.00 & 27.00 & 14.1667 & 4.24332 & 29.95 \\
Km 98 & 30 & 8.00 & 28.00 & 13.0333 & 4.93742 & 37.88 \\
Porv & 30 & 10.00 & 36.00 & 16.3667 & 5.27508 & 32.23 \\
Km 203 & 30 & 6.00 & 28.00 & 12.8000 & 3.97752 & 31.07 \\
\hline
\end{tabular}

- El proceso de pre aclimatación de 4 semanas aplicado a vitroplantas de Uncaria tomentosa (Willd) DC., ha permitido obtener el 100\% de supervivencia con tamaños promedios entre $17.07 \mathrm{~cm} \mathrm{y} 19.53 \mathrm{~cm}$.

- La vigorosidad de las vitroplantas después de la pre aclimatación, expresado en número de hojas promedios (7 y 14 hojas aproximadamente), no ha tenido ninguna incidencia en la mortalidad.

- El desarrollo de las vitroplantas en el periodo de aclimatación en los diferentes substratos expresado en términos de altura, así como la vigorosidad expresada en número de hojas no muestran diferencias significativas.

- A pesar de haberse notado un ligero efecto de menor incremento en el tamaño de las vitroplantas en el substrato IIAP $(2.87 \mathrm{~cm}$.), el crecimiento promedio alcanzado en los cuatro substratos al final de la aclimatación $(20.8-24.8 \mathrm{~cm})$ y el número promedio de hojas $(12$ - 16) han sido adecuados para el desarrollo posterior en condiciones de ambiente natural.

\section{Agradecimientos}

Al Instituto de Biotecnología de la UNALM (IBT), en donde el Autor Principal desarrolló previamente toda la etapa de estandarización de la producción in vitro conjuntamente con la Profesora Lourdes Tapia y Figueroa Directora del IBT. A la UNU por las facilidades en infraestructura recibidos para el desarrollo de la etapa experimental, y al IIAP a través del Ing. Andrés Castillo Quiliano y del Técnico Wilson Saldaña Meléndez, por las facilidades prestadas en el desarrollo de las plantas en vivero posteriores al periodo de aclimatación.

\section{Literatura citada}

Agramonte D., Jiménez F. \& Dita M.A. 1998. Aclimatización. En: Propagación y Mejora de Plantas 
Diciembre 2006

por Biotecnología. (Ed.) Pérez Ponce, J.N. Instituto de Biotecnología de las Plantas Santa Clara. Cuba.

Cuellar E. 1996. Estudio comparativo de propagación por estacas de Uncaria tomentosa (Wild) DC en diferentes condiciones micro ambientales. Tesis Ingeniero Forestal UNALM.

Domínguez G. 1997. Uña de gato y producción sostenible. Universidad Nacional Agraria La Molina. Lima, Perú.

Domínguez G. \& Tapia L. 2000. Reportes de archivo de ensayos de estandarización in vitro de Uncaria tomentosa. Instituto de Biotecnología, Universidad Agraria la Molina, Lima, Perú.

Flores Y. 1995. Propagación por semilla de "uña de gato" (Uncaria tomentosa). Boletín Técnico 5. INIA. Lima.

Instituto de Investigación de la Amazonia Peruana. 1999. Zonificación Ecológica Económica de la región de Ucayali. Pucallpa, Perú.

Luya U. 1999. Micropropagación in vitro y aclimatación de Marigold (Tagetes erecta). Tesis para optar el título de Biólogo, Universidad Agraria La Molina. Lima, Perú.

Piñan A. 1995. La uña de gato en Tingo María. En: Prensa UNASINA Vol. 2 (14). Tingo María, Perú.

Preece J.E. \& Sutter E.G. 1991. Aclimatizacion of micropropagated plant to the greenhouse and field. En:
Debergh, P.C. \& Zimmerman, R.H. Micropropagation thecnology and application (Ed) Dordrech Kluwer Academic Press. : 71-93.

Reigosa M., Pedrol N. \& Sánchez A. 2004. La Eco fisiología Vegetal una Ciencia de Síntesis: Cap. 34 Eco fisiología del cultivo in vitro: Aclimatación de plantas. Thomson Ed. España. : 1017-1053.

Roca W. 1991. Cultivo de tejidos en la agricultura: Fundamentos y aplicación. CIAT, Colombia.

Sotolongo R. 2000. Micropropagación de Psidium salutare (HBK) Berg. Tesis presentada en opción al grado científico de Doctor en Ciencias Forestales. Pinar del Río, Cuba.

Texeira J.B, Lemos J.I. \& Coelhe M.C. 1995. Micropropagacao de especies lenhosas da mata atlántica. En: Congreso Brasileiro de Fisiología Vagetal. 5. (Ed) Lauras Anais.

Ziv M. 1991. Vitrification: Morphological and physiological disorders of in vitro plant. En Debergh, P.C. Zimmerman, R.H. 1991. Micropropagation: Technology and application. (Ed.) Dordrecht. Kluwer Academic. : 49 -69 .

\footnotetext{
${ }^{1}$ Universidad Nacional Agraria La Molina, Av La Universidad s/n-La Molina, Lima Perú. gdominguez@lamolina.edu.pe

${ }^{2}$ Universidad Nacional de Ucayali. Av. Federico Basadre Km 6, Pucallpa, Ucayali Perú. luzdonayre@yahoo.es
} 